\title{
Normal domains with monomial presentations
}

\author{
Isabel Goffa, Eric Jespers, Jan Okniński
}

\begin{abstract}
Let $A$ be a finitely generated commutative algebra over a field $K$ with a presentation $A=K\left\langle X_{1}, \ldots, X_{n} \mid R\right\rangle$, where $R$ is a set of monomial relations in the generators $X_{1}, \ldots, X_{n}$. So $A=K[S]$, the semigroup algebra of the monoid $S=\left\langle X_{1}, \ldots, X_{n} \mid R\right\rangle$. We characterize, purely in terms of the defining relations, when $A$ is an integrally closed domain, provided $R$ contains at most two relations. Also the class group of such algebras $A$ is calculated.
\end{abstract}

Mathematics Subject Classification 2000: primary 16S36, 13B22; secondary 14M25, 16H05, 13C20, 20M14

keywords: normal domain, class group, finitely presented algebra, semigroup algebra, commutative semigroup, normal semigroup

\section{Introduction}

Normal Noetherian domains, also called integrally closed Noetherian domains, are of fundamental importance in several areas of mathematics. In the literature one can find several concrete constructions of such rings that are algebras over a field $K$ and that have a presentation in which the relations are of monomial type. Such algebras are commutative semigroup algebras $K[S]$ of a finitely generated abelian and cancellative monoid $S$ (that is, $S$ is a submonoid of a finitely generated abelian group $G$ ). Within the context of commutative ring theory, these algebras received a lot of attention (see for example [2, 9]). We recall some well known facts. First, a commutative semigroup algebra $K[S]$ of a monoid $S$ is Noetherian if and only if $S$ is finitely generated. In this case $K[S]$ also is finitely presented. Second, $K[S]$ is a domain if and only if $S$ is a submonoid of a torsion free abelian group. Recall that an affine semigroup $S$ is a finitely generated submonoid of a free abelian group. If, moreover, the unit groups $\mathrm{U}(S)$ is trivial, that is $\mathrm{U}(S)=\{1\}$, then $S$ is said to be positive. Third (see 2, Proposition 6.1.4] or [17, Proposition 13.5]), if $M$ is an affine monoid then $K[M]$ is normal if and only if $M$ is normal (i.e. if $g \in M M^{-1}$, the group of fractions of $M$, and $g^{n} \in M$ for some $n \geq 1$ then $\left.g \in M\right)$. Moreover, such monoids $M$ are precisely the monoids of the form $U \times M^{\prime}$, where $U$ is a finitely generated free abelian group and $M^{\prime}$ is a positive monoid so that $M^{\prime}=\left(M^{\prime}\right)\left(M^{\prime}\right)^{-1} \cap F^{+}$, with $F^{+}$the positive cone of a free abelian group $F$. Note that if $M$ is positive and of rank $n$, that is $M M^{-1}$ is a group of 
torsion free rank $n$, then $M$ is isomorphic to a submonoid of $\mathbb{N}^{n}$, a free abelian monoid of rank $n$. So, normality of $K[S]$ is a homogeneous property, i.e., a condition on the monoid $S$. This was one of the motivating reasons for these investigations. Furthermore, it is well known that $\operatorname{cl}(K[S])$, the class group of $K[S]$, is naturally isomorphic with $\operatorname{cl}(S)$, the class group of $S$ (see for example [1. Theorem 2.3.1]). As an application one obtains much easier calculations for the class group of several classical examples of Noetherian normal domains. So the study of normal positive monoids is relevant in the context of number theory. Another reason for their importance is their connection to geometry, especially in the context of toric varieties and convex polytopes (see for example [1, 13, 17, 18, for an extensive bibliography of the subject, its computational aspects and applications to other fields).

The study of the above problems is also crucial in a noncommutative setting. Indeed, noncommutative maximal orders of the form $K[S]$, with $S$ a cancellative nonabelian monoid, appear in the search of set-theoretical solutions of the quantum Yang-Baxter equation. Gateva-Ivanova and Van den Bergh [8] and Etingof, Schedler and Soloviev in [6] showed that such solutions are determined by monoids $M$ of $I$-type. In [10] this was extended to the larger class of monoids of $I G$-type. Such monoids are contained in a finitely generated abelian-by-finite group and their algebras share many properties with commutative polynomial algebras. In particular, they are maximal orders in a division algebra and the algebraic structure of $M$ is determined by a normal positive submonoid and a finite solvable group acting on it. More generally, as shown in 11, every prime maximal order $K[S]$ satisfying a polynomial identity is in some sense built on the basis of a normal abelian submonoid of $S$ and every abelian normal monoid can be used to construct a family of noncommutative maximal orders. For more details on noncommutative orders we refer the reader to 12 .

In this paper we deal with Noetherian commutative semigroup algebras $K[S]$ that are defined by at most two monomial relations. We obtain a characterization purely in terms of the defining relations, of when such an algebra is a normal domain. It is easily seen that if $K[S]$ is such an algebra then $S$ has codimension at most 2. Recall that $S$ has codimension $n-d$ if it is generated by $n$ elements and $S \subseteq \mathbb{N}^{d}$. Recently Dueck, Hoşten and Sturmfels obtained necessary conditions for such algebras to be normal. In order to state this we recall that given a term order $\prec$ on the free abelian monoid $F=\left\langle u_{1}, \ldots, u_{n}\right\rangle$, the initial ideal $I_{\prec}$ of $S$ (corresponding to this order) is the ideal of $F$ consisting of all leading (highest) monomials in every relation that holds in $S$.

Proposition 1.1 ([5, Theorem 1]) Suppose $S$ is a positive monoid of codimension two. If $S$ is normal then $S$ has a square free initial ideal (that is, a semiprime ideal in $S$ ).

If, moreover, $S$ is a homogeneous monoid (that is, $S$ is defined by relations that are homogeneous with respect to the total degree) then the converse follows from Proposition 13.15 in [17. The latter says that if $S$ is a homogeneous submonoid of $\mathbb{Z}^{d}$ such that for some order $\prec$ the corresponding initial ideal $I_{\prec}$ 
is square free, then $S$ is a normal monoid. Theorem 2 in [5] also says that if $S$ is a positive monoid of codimension $n-d$ then there is an algorithm to decide whether $A$ is normal, whose running time is polynomial.

From the characterization proved in this paper it follows that the converse of Proposition 1.1 holds for an arbitrary positive monoid $S$ defined by at most two relations (so without the homogeneous assumption). Exercise 13.17 in [17] implies that this converse is false in general. It is worth mentioning that other constraints for normality of abelian monoids have been studied in [14, 15, 16].

As an application, we determine the class group $\operatorname{cl}(S)$ in terms of the combinatorial data contained in the defining relations.

\section{One-relator monoids}

Our main aim is to describe when a positive monoid defined by at most two relations is normal. A first important obstacle to overcome is to determine when such monoids are cancellative, i.e., when they are contained in a group and next to decide when this group can be assumed torsion free. Because of the comments given in the introduction, and since we are mainly interested in such monoids that are normal, we only need to deal with monoids $S$ so that $\mathrm{U}(S)=\{1\}$. In this context we mention that in [3] an algorithm of Contejean and Devie is used to determine whether a finitely generated monoid given by a presentation is cancellative.

We will use the following notation. By $\mathrm{FaM}_{n}$ we denote a free abelian monoid of rank $n$. If $\mathrm{FaM}_{n}=\left\langle u_{1}, \ldots, u_{n}\right\rangle$ and $w=u_{1}^{a_{1}} \cdots u_{n}^{a_{n}} \in \mathrm{FaM}_{n}$, then $\operatorname{put} \operatorname{supp}(w)=\left\{u_{i} \mid a_{i} \neq 0\right\}$, the support of $w$, and $\operatorname{Hsupp}(w)=\left\{u_{j} \mid a_{j}>1\right\}$. We say that $w$ is square free if $\operatorname{Hsupp}(w)=\emptyset$. Now, suppose $S$ has a presentation

$$
S=\left\langle u_{1}, \ldots, u_{n} \mid w_{1}=v_{1}, \ldots, w_{m}=v_{m}\right\rangle,
$$

where $w_{i}, v_{i}$ are nonempty words in the free abelian monoid $\mathrm{FaM}_{n}=\left\langle u_{1}, \ldots, u_{n}\right\rangle$. Clearly, $\mathrm{U}(S)=\{1\}$ and if $S$ is cancellative, then we may assume it has a presentation with

$$
\operatorname{supp}\left(w_{i}\right) \cap \operatorname{supp}\left(v_{i}\right)=\emptyset,
$$

for all $i$.

Recall from Lemma 6.1 in [14 that if $K[S]$ is a normal domain and $w_{i}=v_{i}$ is independent of the other defining relations then at least one of $w_{i}$ or $v_{i}$ is square free.

Proposition 2.1 Let $S$ be an abelian monoid defined by the presentation

$$
\left\langle u_{1}, \ldots, u_{n} \mid u_{1} \cdots u_{k}=u_{k+1}^{a_{k+1}} \cdots u_{n}^{a_{n}}\right\rangle
$$

for some positive integers $a_{k+1}, \ldots, a_{n}$ and some $k<n$. Let $\operatorname{FaM}_{k(n-k)}=\left\langle x_{i, j}\right|$ $1 \leq i \leq k, 1 \leq j \leq n-k\rangle$, a free abelian monoid of rank $k(n-k)$. For $1 \leq j \leq k$ put

$$
v_{j}=x_{j, 1}^{a_{k+1}} x_{j, 2}^{a_{k+2}} \cdots x_{j, n-k}^{a_{n}},
$$


and for $k+1 \leq j \leq n$ put

$$
v_{j}=x_{1, j-k} x_{2, j-k} \cdots x_{k, j-k} .
$$

Then $S \cong V=\left\langle v_{1}, \ldots, v_{n}\right\rangle \subseteq \operatorname{FaM}_{k(n-k)}$ (in particular, $S$ is cancellative) and $\left\langle v_{1}, \ldots, v_{n}\right\rangle$ has $v_{1} \cdots v_{k}=v_{k+1}^{a_{k+1}} \cdots v_{n}^{a_{n}}$ as its only defining relation.

Proof. Let $V=\left\langle v_{1}, \ldots, v_{n}\right\rangle \subseteq \operatorname{FaM}_{k(n-k)}$. Clearly, $v_{1} \cdots v_{k}=v_{k+1}^{a_{k+1}} \cdots v_{n}^{a_{n}}$ and thus $V=\left\langle v_{1}, \ldots, v_{n}\right\rangle$ is a natural homomorphic image of $S$.

Since all $a_{i} \neq 0$, it is easy to see that every relation in $V$ (with disjoint supports with respect to the $v_{i}$ 's) must involve all generators $v_{i}$. Moreover, since $v_{1}, v_{k+1}$ are the only generators involving $x_{1,1}$, it follows that in such a relation $v_{1}, v_{k+1}$ are on opposite sides of the equality. And also $v_{k+2}, \ldots, v_{n}$ must be on the side opposite to $v_{1}$ (look at the appearance of $x_{1,2}, x_{1,3}, \ldots, x_{1, n-k}$ in order to see this). Similarly, by looking at the appearance of $x_{21}, x_{31}, \ldots, x_{k 1}$, we get that $v_{2}, \ldots, v_{k}$ must be on the side opposite to $v_{k+1}$. It follows that every relation in $V$, possibly after cancellation, must be of the form

$$
v_{1}^{c_{1}} \cdots v_{k}^{c_{k}}=v_{k+1}^{c_{k+1}} \cdots v_{n}^{c_{n}}
$$

for some positive integers $c_{j}$. Again, using the fact that $x_{i, j}$ 's are independent and comparing the exponent of $x_{i, j}$ on both sides of (11), we get that $a_{k+j} c_{i}=$ $c_{k+j}$ for $1 \leq i \leq k$ and $j=1,2, \ldots, n-k$. This implies that $c_{1}=c_{2}=\cdots=c_{k}$. Hence relation (11) is of the form $\left(v_{1} \cdots v_{k}\right)^{c_{1}}=\left(v_{k+1}^{a_{k+1}} \cdots v_{n}^{a_{n}}\right)^{c_{1}}$. So it is a consequence of the relation defining $S$ with every $u_{j}$ replaced by $v_{j}$. It follows that $V \cong S$.

Note that one can verify that the monoid $V$, as described in the previous proposition, is such that $V=V V^{-1} \cap \mathrm{FaM}_{k(n-k)}$. So, by the comments given in the introduction, $V$ is normal. Alternatively, it easily follows from the defining relation that $S=\bigcup_{1 \leq i \leq k} F_{i}$, with $F_{i}=\left\langle u_{j} \mid 1 \leq j \leq n, j \neq i\right\rangle$ a free abelian monoid with group of quotients $S S^{-1}$. Since each $F_{i}$ is normal we thus obtain that $S$ is normal as well ([1, Proposition 3.1.1]).

Hence, the Proposition 2.1 and its preceding comment yield at once a description one-relator positive monoids that are normal.

Proposition 2.2 Let $S$ be the abelian monoid defined by the presentation

$$
\left\langle u_{1}, \ldots, u_{n} \mid w_{1}=w_{2}\right\rangle,
$$

with nonempty words $w_{1}=u_{1}^{a_{1}} \cdots u_{k}^{a_{k}}, w_{2}=u_{k+1}^{a_{k+1}} \cdots u_{n}^{a_{n}}$, where $k<n$, and each $a_{i}$ is a nonnegative integer. The following conditions are equivalent.

1. The semigroup $S$ is a normal positive monoid, normal (or equivalently, the semigroup algebra $K[S]$ is a normal domain).

2. $\operatorname{Hupp}\left(w_{1}\right)=\emptyset$ or $\operatorname{Hsupp}\left(w_{2}\right)=\emptyset$. 
In the remainder of this section we describe the class group $\operatorname{cl}(S)$ of a onerelator normal positive monoid $S$. For convenience sake we recall some terminology for an affine normal monoid $M$ (see [4, 9]; at an algebra level we refer to [7). For a subset $I$ of $M M^{-1}$ we put $(M: I)=\left\{g \in M M^{-1} \mid g I \subseteq M\right\}$. A fractional ideal $I$ of $M$ is a subset of $M M^{-1}$ so that $M I \subseteq I$ and $m I \subseteq M$ for some $m \in M$. A fractional ideal is said to be divisorial if $I=I^{*}$, where $I^{*}=(M:(M: I))$. The set of all divisorial fractional ideals is denoted by $D(M)$. It is a free abelian group for the divisorial product $I * J=(I J)^{*}$, for $I, J \in D(M)$, with basis the set of minimal prime ideals. Also, $M=\bigcap M_{P}$, where the intersection runs over all minimal primes of $M$, and all localizations $M_{P}$ are discrete valuation monoids (see for example [4, 9]). Furthermore, for an ideal $I$ of $M$ one has, in the divisorial group $D(M)$, that $I=\left(\prod_{P} P^{n(P)}\right)^{*}$ if and only if $M_{P} I=M_{P} P^{n(P)}$, with all $n_{P} \geq 0$. Moreover, $n_{P}>0$ if and only if $I \subseteq P$.

By definition $\operatorname{cl}(M)=D(M) / P(M)$, where $P(M)=\left\{M g \mid g \in M M^{-1}\right\}$.

Let $S$ be again as in Proposition 2.2. We will use the same notation for the generators $u_{i}$ of the free monoid $\mathrm{FaM}_{n}$ and for their images in $S$, if unambiguous. So, every $S_{u_{j}}$ in $D(S)$ is a (unique) product of the minimal primes of $S$. In the following lemma we compute these decompositions provided all $a_{i}$ are positive integers. Clearly, in this case, the minimal primes of $S$ are the ideals $P_{y z}$ generated by the set $\left\{u_{y}, u_{z}\right\}$, where $y \in\{1, \ldots, k\}, z \in\{k+1, \ldots, n\}$.

Lemma 2.3 Let $S=\left\langle u_{1}, \ldots, u_{n} \mid u_{1} \cdots u_{k}=u_{k+1}^{a_{k+1}} \cdots u_{n}^{a_{n}}\right\rangle$ be a normal monoid, with all $a_{i} \geq 1$, and let $P_{y z}$ denote the minimal prime ideal of $S$ that is generated by the set $\left\{u_{y}, u_{z}\right\}$, where $y \in\{1, \ldots, k\}, z \in\{k+1, \ldots, n\}$. Then

$$
S u_{z}=P_{1 z} * \cdots * P_{k z} \quad \text { and } S u_{y}=P_{y k+1}^{a_{k+1}} * \cdots * P_{y n}^{a_{n}},
$$

for $z \in\{k+1, \ldots, n\}$ and $y \in\{1, \ldots, k\}$.

Proof. First, let $y \in\{1, \ldots, k\}$. Note that the only minimal primes containing $u_{y}$ are $P_{y, z}$, with $z \in\{k+1, \ldots, n\}$. Hence $S u_{y}=\left(\prod_{k \leq z \leq n} P_{y, z}^{e(z)}\right)^{*}$, with $e(z) \geq 1$. Furthermore, in the localization $T=S_{P_{y, z}}$ we have that $u_{i}, u_{j}$ are invertible for $y \neq i \in\{1, \ldots, k\}$ and $z \neq j \in\{k+1, \ldots, n\}$. Hence, from the defining relation it follows that $T u_{y}=T u_{z}^{a_{z}}$ and thus also $T u_{y}=T P_{y, z}^{a_{z}}$. Consequently, $e(z)=a_{z}$ and thus $S u_{y}=P_{y k+1}^{a_{k+1}} * \cdots * P_{y n}^{a_{n}}$, as desired.

Second, assume $z \in\{k+1, \ldots, n\}$. Then, for any $y \in\{1, \ldots, k\}$, it is easily seen from the defining relation that $T u_{y} \subseteq T u_{z}$, with $T=S_{P_{y, z}}$. Thus $T u_{z}=T P_{y, z}$. Therefore, as above, $S u_{z}=P_{1 z} * \cdots * P_{k z}$.

Theorem 2.4 Let $S=\left\langle u_{1}, \ldots, u_{n}, \ldots, u_{m} \mid u_{1} \cdots u_{k}=u_{k+1}^{a_{k+1}} \cdots u_{n}^{a_{n}}\right\rangle$ be $a$ positive normal monoid (with all $a_{i} \geq 1$ and $n \leq m$ ). Then

$$
\operatorname{cl}(K[S]) \cong \operatorname{cl}(S) \cong \mathbb{Z}^{k(n-k)-(n-1)} \times\left(\mathbb{Z}_{d}\right)^{k-1},
$$

where $d=\operatorname{gcd}\left(a_{k+1}, \ldots, a_{n}\right), k(n-k)$ is the number of minimal primes in $S$ not containing one of the independent generators $u_{n+1}, \ldots, u_{m}$, and $m-1$ is the torsion free rank of $S S^{-1}$. 
Proof. Clearly, $S=S^{\prime} \times \mathrm{FaM}_{m-n}$, where $\mathrm{FaM}_{r}=\left\langle a_{n+1}, \ldots, a_{m}\right\rangle$ is a free abelian monoid, and $S^{\prime}=\left\langle u_{1}, \ldots, u_{n} \mid u_{1} \cdots u_{k}=u_{k+1}^{a_{k+1}} \cdots u_{n}^{a_{n}}\right\rangle$. So, $S$ is normal if and only if $S^{\prime}$ is normal. Because also $\operatorname{cl}\left(S^{\prime}\right)=\operatorname{cl}(S)$, we may assume $S=S^{\prime}$.

Clearly, the result is true for $k=1$. So assume that $k \geq 2$. As there are $k(n-k)$ minimal primes $P_{y z}$ in $S$ (with $1 \leq y \leq k, k+1 \leq z \leq n$ ), we get that $D(S) \cong \mathbb{Z}^{k(n-k)}$. On the other hand, $P(S)=\operatorname{gr}\left(S u_{i} \mid i=1, \ldots, n\right)$. By Lemma 2.3, $S u_{j}=\left(\prod_{l=1}^{k} P_{l j}\right)^{*}, S u_{i}=\left(\prod_{l=k+1}^{n} P_{i l}^{a_{l}}\right)^{*}$ for $i \in\{1, \ldots, k\}, j \in$ $\{k+1, \ldots, n\}$. We consider

$$
\operatorname{cl}(S)=\operatorname{gr}\left(P_{y z} \mid y \in\{1, \ldots, k\}, z \in\{k+1, \ldots, n\}\right) / \operatorname{gr}\left(S u_{i} \mid 1 \leq i \leq n\right) .
$$

as a finitely generated $\mathbb{Z}$-module. So its presentation corresponds to an integer matrix $M$ of size $k(n-k) \times n$. The rows of $M$ are indexed by elements of the set $R=\{(i, j) \mid i \in\{1, \ldots, k\}, j \in\{k+1, \ldots, n\}\}$. We agree on the lexicographic ordering of the set of rows of $M$. The columns are indexed by $C=\{1,2, \ldots, n\}$, where the $i$-th column corresponds to the generator $S u_{i}$, written as a vector in terms of the minimal primes of $S$.

We consider the block decomposition of $M$ determined by the following partitions of the sets $C$ and $R$ of columns and rows: $C=D_{1} \cup D_{2}$, where $D_{1}=\{1, \ldots, k\}$ and $D_{2}=\{k+1, \ldots, n\}$ and $R=R_{1} \cup \cdots \cup R_{k}$, where $R_{i}=$ $\{(i, j) \mid j=k+1, \ldots, n\}$. Then $M$ has the following form:

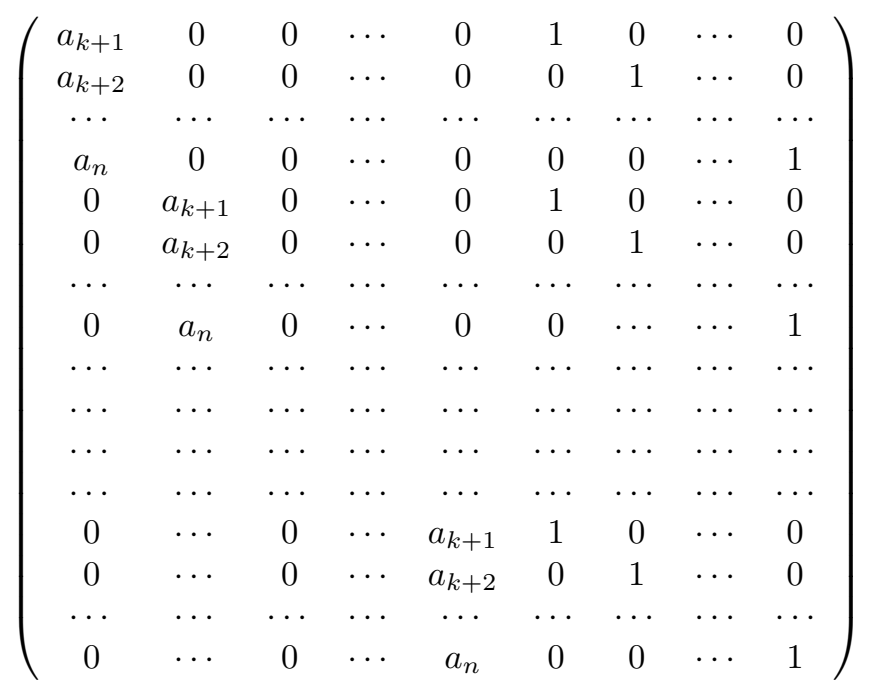

We subtract the subsequent rows of the last row block $R_{k}$ from the corresponding rows of all other row blocks. Then from column $k$ we subtract $\sum_{i=k+1}^{n} a_{i} C_{i}$, where $C_{i}$ denotes the $i$-the column. The obtained matrix $M^{\prime}$ has the $\left(R_{k}, C\right)$ block of the form $M_{R_{k}, C}^{\prime}=(0, I)$, where $I$ is the $(n-k) \times(n-k)$ identity matrix and $M_{R_{i} D_{2}}^{\prime}$ is a zero matrix for every $i \neq k$. Let $T=R \backslash R_{k}$. The last column of the submatrix $M_{T, D_{1}}^{\prime}$ has the form $\left(-a_{k+1}, \ldots,-a_{n}, \ldots,-a_{k+1}, \ldots,-a_{n}\right)^{t}$, 
hence adding all other columns of $M_{T, D_{1}}^{\prime}$ to it, we get a matrix $N$ such that $N=M_{T, D_{1}}$. Clearly, the normal form of $N$ involves $k-1$ entries equal to $d=\operatorname{gcd}\left(a_{k+1}, \ldots, a_{n}\right)$ and no other nonzero entries. The result follows.

\section{Two-relator monoids}

In this section we obtain a characterization of normal positive monoids that are defined by two relations. The class group of such monoids $S$, and therefore of the corresponding algebras $K[S]$, is also determined.

Theorem 3.1 Let $S=\left\langle u_{1}, \ldots, u_{n}\right\rangle$ be a finitely presented abelian monoid with independent defining relations $w_{1}=w_{2}$ and $w_{3}=w_{4}$ and, $\left|\operatorname{supp}\left(w_{i}\right)\right| \geq 1$ for all $i$. The following conditions are equivalent.

1. The semigroup $S$ is a normal positive monoid (or equivalently, the semigroup algebra $K[S]$ is a normal domain).

2. $S$ is a positive monoid with an initial ideal $I_{\prec}$ of $S$ that is square free.

3. The following conditions hold:

(a) $\operatorname{supp}\left(w_{1}\right) \cap \operatorname{supp}\left(w_{2}\right)=\emptyset, \operatorname{supp}\left(w_{3}\right) \cap \operatorname{supp}\left(w_{4}\right)=\emptyset$,

(b) $\operatorname{Hsupp}\left(w_{1}\right)=\emptyset$ or $\operatorname{Hsupp}\left(w_{2}\right)=\emptyset$,

(c) $\operatorname{Hsupp}\left(w_{3}\right)=\emptyset$ or $\operatorname{Hsupp}\left(w_{4}\right)=\emptyset$,

(d) if there exist $i \in\{1,2\}, j \in\{3,4\}$ such that $\operatorname{supp}\left(w_{i}\right) \cap \operatorname{supp}\left(w_{j}\right) \neq$ $\emptyset$, then one of the following properties holds (we may assume for simplicity that $i=1$ and $j=3$ ):

- $\operatorname{supp}\left(w_{k}\right) \cap \operatorname{supp}\left(w_{l}\right)=\emptyset$ for all pairs $\{k, l\} \neq\{1,3\}$ with $k \neq l$, and $\operatorname{Hsupp}\left(w_{2}\right)=\emptyset$ or $\operatorname{Hsupp}\left(w_{4}\right)=\emptyset$,

- there exists a pair $k \neq l$ such that $\{2,4\} \neq\{k, l\} \neq\{1,3\}$ and $\operatorname{supp}\left(w_{k}\right) \cap \operatorname{supp}\left(w_{l}\right) \neq \emptyset$ (for simplicity assume $k=2, l=3$ ), $\operatorname{supp}\left(w_{4}\right) \cap \operatorname{supp}\left(w_{i}\right)=\emptyset$ for $i=1,2,3$ and $\operatorname{Hsupp}\left(w_{4}\right)=\emptyset$.

Proof. Note that $S=S_{1} \times S_{2}$, where $S_{2}$ is the free abelian monoid generated by

$$
\left\{u_{1}, \ldots, u_{n}\right\} \backslash\left(\bigcup_{i=1}^{4} \operatorname{supp}\left(w_{i}\right)\right)
$$

and

$$
S_{1}=\left\langle\bigcup_{i=1}^{4} \operatorname{supp}\left(w_{i}\right)\right\rangle .
$$

Since $S_{2}$ is a normal positive monoid, it follows that $S$ is a normal positive monoid if and only if $S_{1}$ is such a monoid, i.e. we may assume that $\left\{u_{1}, \ldots, u_{n}\right\}=\bigcup_{i=1}^{4} \operatorname{supp}\left(w_{i}\right)$. 
It follows from Proposition 1.1 that (1) implies (2). We now prove (2) implies (3). So assume that $I=I_{\prec}$ is a square free ideal for some term order $\prec$ and $S$ is a positive monoid. In order to prove (3.a) suppose for example that $\operatorname{supp}\left(w_{1}\right) \cap \operatorname{supp}\left(w_{2}\right) \neq \emptyset$. Then write $w_{1}=u w_{1}^{\prime}, w_{2}=u w_{2}^{\prime}$ for a nontrivial word $u$ and some $w_{1}^{\prime}, w_{2}^{\prime}$ such that $\operatorname{supp}\left(w_{1}^{\prime}\right) \cap \operatorname{supp}\left(w_{2}\right)^{\prime}=\emptyset$. Hence, in $S$, we have $w_{1}^{\prime}=w_{2}^{\prime}$, and thus each of $w_{1}^{\prime}, w_{2}^{\prime}$ is divisible by some of the $w_{j}$ 's. So, by symmetry, we may assume that $w_{1}^{\prime}=w_{3} z$ and $w_{2}^{\prime}=w_{4} y$. Let $m$ be the maximal positive integer such that $w_{1}^{\prime}=w_{3}^{m} z^{\prime}$ and $w_{2}^{\prime}=w_{4}^{\prime} y^{\prime}$ for some $z^{\prime}, y^{\prime}$. Then $z^{\prime}=y^{\prime}$ holds in $S$ and it follows that $z^{\prime}=y^{\prime}$ as words (otherwise $y^{\prime}, z^{\prime}$ would be again divisible by $w_{3}, w_{4}$, respectively, contradicting the choice of $m$ ). It follows that the relation $w_{1}=w_{2}$ is a consequence of $w_{3}=w_{4}$, a contradiction. So (3.a) follows.

In order to prove conditions (3.b),(3.c) and (3.d) we introduce the following notation. For a word $w$ in $u_{1}, \ldots, u_{n}$ we define $\sqrt{w}=x_{1} \cdots x_{p}$ where $\operatorname{supp}(w)=$ $\left\{x_{1}, \ldots, x_{p}\right\}$.

Note that if $\operatorname{supp}\left(w_{1}\right) \cap \operatorname{supp}\left(w_{3}\right) \neq \emptyset$ then we must have that $\operatorname{supp}\left(w_{2}\right) \cap$ $\operatorname{supp}\left(w_{4}\right)=\emptyset$. Indeed, for otherwise, the ideal $K[S]\left(w_{1}-w_{2}, w_{3}-w_{4}\right) \subseteq$ $K[S]\left(u_{i}, u_{j}\right)$, for some $i \neq j$. Since both ideals are height two primes, they must be equal, a contradiction (note that $S$ is, by assumption, a positive monoid and thus $K[S]$ is a domain). If $\operatorname{supp}\left(w_{2}\right) \cap \operatorname{supp}\left(w_{3}\right) \neq \emptyset$ then, by the same reasoning, $\operatorname{supp}\left(w_{1}\right) \cap \operatorname{supp}\left(w_{4}\right)=\emptyset$. Hence we have shown that either all $\operatorname{supp}\left(w_{i}\right)$ are disjoint or $\operatorname{supp}\left(w_{i}\right) \cap \operatorname{supp}\left(w_{j}\right) \neq \emptyset$ for exactly one pair $i, j$ or this holds for exactly two pairs and these pairs are of the form $i, j$ and $i, m$ for some $i, j, m$. So, by symmetry, it is enough to deal with the three cases considered below.

If all $\operatorname{supp}\left(w_{i}\right)$ are disjoint then let for example $w_{2} \prec w_{1}$ and $w_{4} \prec w_{3}$. It easily follows from the assumption that $w_{1}, w_{3}$ must be square free and hence (3.b),(3.c) and (3.d) hold.

Next, assume that $\operatorname{supp}\left(w_{1}\right) \cap \operatorname{supp}\left(w_{3}\right) \neq \emptyset$ and $\operatorname{supp}\left(w_{i}\right) \cap \operatorname{supp}\left(w_{j}\right)=\emptyset$ for every pair $(i, j) \neq(1,3)$.

To prove (3.d) we need to show that $\operatorname{Hsupp}\left(w_{i}\right)=\emptyset$ for $i=2$ or $i=4$. So, suppose otherwise, that is, $w_{2}, w_{4}$ are not square free. Then $w_{1}, w_{3} \in I$ and $w_{2} \prec w_{1}, w_{4} \prec w_{3}$ (because for example if $w_{1} \prec w_{2}$ then $w_{2} \in I$, so $w_{2} \neq \sqrt{w_{2}} \in I$, whence $\sqrt{w_{2}}$ is in a nontrivial relation in $S$, but it cannot be divisible by any of the words $w_{i}, i=1,2,3,4$, a contradiction). Let $w_{k}=w w_{k}^{\prime}$ for $k=1,3$, where $\operatorname{supp}\left(w_{1}^{\prime}\right) \cap \operatorname{supp}\left(w_{3}^{\prime}\right)=\emptyset$. Then $w_{3} w_{1}^{\prime}=w_{1} w_{3}^{\prime}$ as words and, in $S$, we have $w_{3} w_{1}^{\prime}=w_{4} w_{1}^{\prime}$ and $w_{1} w_{3}^{\prime}=w_{2} w_{3}^{\prime}$. So one of the words $w_{4} w_{1}^{\prime}, w_{2} w_{3}^{\prime}$ is in $I$. Therefore $\sqrt{w_{4}} w_{1}^{\prime} \in I$ or $\sqrt{w_{2}} w_{3}^{\prime} \in I$. Say, for example, that the former holds. Then $\sqrt{w_{4}} w_{1}^{\prime}$ is in a nontrivial relation in $S$. But it is easy to see that $\sqrt{w_{4}} w_{1}^{\prime}$ cannot have $w_{i}$ as a subword for every $i=1,2,3,4$. This contradiction establishes assertion (3.d).

To prove (3.b) and (3.c) in this case, suppose for example that $\operatorname{Hsupp}\left(w_{2}\right)=$ $\emptyset$ and $\operatorname{Hsupp}\left(w_{3}\right) \neq \emptyset \neq \operatorname{Hsupp}\left(w_{4}\right)$. An argument as before shows that $w_{4} \prec$ $w_{3} \in I$ and $w_{4} \notin I$. Hence $w_{3} \neq \sqrt{w_{3}} \in I$. Then $\sqrt{w_{3}}=w_{1} x$ for a word $x$. The only relation in which $w_{1} x$ can occur must be of the form $w_{1} x=w_{2} x$, whence we have $w_{2} \prec w_{1}$. Write $w_{3}=v_{1} v_{3}$ where $\operatorname{supp}\left(v_{1}\right)=\operatorname{supp}\left(w_{1}\right)$ and $\operatorname{supp}\left(v_{3}\right) \cap \operatorname{supp}\left(w_{1}\right)=\emptyset$. Let $k \geq 1$ be minimal such that $w_{3}$ divides $w_{1}^{k} v_{3}$. 
Then $w_{1}^{k} v_{3}=w_{3} y$ for a subword $y$ of $w_{1}$ such that $y \neq w_{1}$. So, in $S$, we get $w_{2}^{k} v_{3}=w_{1}^{k} v_{3}=w_{3} y=w_{4} y$. Since the word $w_{4} y$ is not divisible by $w_{1}, w_{2}, w_{3}$ and $w_{4} \neq \sqrt{w_{4}}$, it follows that $\sqrt{w_{4} y} \notin I$, whence $w_{2}^{k} v_{3} \in I$. Then $w_{2} v_{3} \in I$. But the only relation containing this word is $w_{2} v_{3}=w_{1} v_{3}$. Since $w_{2} v_{3} \prec w_{1} v_{3}$, we get a contradiction. We have shown that (3.b),(3.c) are satisfied.

Finally, consider the case where there are at exactly two overlaps between the supports of $w_{i}, i=1,2,3,4$. We may assume that $\operatorname{supp}\left(w_{1}\right) \cap \operatorname{supp}\left(w_{3}\right) \neq \emptyset$ and $\operatorname{supp}\left(w_{2}\right) \cap \operatorname{supp}\left(w_{3}\right) \neq \emptyset$. $\operatorname{So} \operatorname{supp}\left(w_{4}\right) \cap \operatorname{supp}\left(w_{j}\right)=\emptyset$ for every $j \neq 4$.

Suppose that $\operatorname{Hsupp}\left(w_{4}\right) \neq \emptyset$. Let $w_{1}=a b, w_{2}=c d, w_{3}=a^{\prime} c^{\prime} e$, where $\operatorname{supp}(a)=\operatorname{supp}\left(a^{\prime}\right), \operatorname{supp}(c)=\operatorname{supp}\left(c^{\prime}\right)$ and the remaining factors have pairwise disjoint supports. Let $a_{0}, a_{0}^{\prime}$ be words of minimal length such that $a a_{0}=a^{\prime} a_{0}^{\prime}$. Clearly, $a_{0}^{\prime}$ is not divisible by $a$ and $\operatorname{supp}\left(a_{0}^{\prime}\right) \cap \operatorname{supp}\left(a_{0}\right)=\emptyset$.

Now $a b c^{\prime} e=c d c^{\prime} e$ in $S$ and $a^{\prime} c^{\prime} e b=w_{4} b$ in $S$. So $c d c^{\prime} e a_{0}=w_{4} b a_{0}^{\prime}$ in $S$ and hence one of these words is in $I$. If $w_{4} b a_{0}^{\prime}$ is in $I$ then $\sqrt{w_{4} b a_{0}^{\prime}} \in I$, which is not possible because $\sqrt{w_{4} b a_{0}^{\prime}}$ cannot be rewritten in $S$ (as $\sqrt{w_{4}}$ is a proper subword of $w_{4}$ with support independent of $w_{1}, w_{2}, w_{3}$ and $b a_{0}^{\prime}$ is not divisible by any of $\left.w_{1}, w_{2}, w_{3}\right)$. Hence $c d c^{\prime} e a_{0} \in I$. Then $c d e a_{0} \in I$ because $I$ is square free. But the only way to rewrite $c d e a_{0}$ in $S$ is $c d e a_{0}=a b e a_{0}$. Hence $a b e a_{0} \prec c d e a_{0}$, so also $w_{1}=a b \prec c d=w_{2}$. However, repeating the above argument with the roles of $w_{1}$ and $w_{2}$ switched, we also get $w_{2} \prec w_{1}$, a contradiction. We have proved that $w_{4}$ is square free, so (3.d) holds, and (3.c) also holds.

It remains to prove condition (3.b). Suppose that $w_{1}, w_{2}$ are not square free. By symmetry, we may assume that $w_{2} \in I$. Then $\sqrt{w_{1}} \in I$ and in particular the word $\sqrt{w_{1}}$ it must be divisible by $w_{3}$. $\operatorname{But} \operatorname{supp}\left(w_{2}\right) \cap \operatorname{supp}\left(w_{3}\right) \neq \emptyset$ by the assumption, so $\operatorname{supp}\left(w_{2}\right) \cap \operatorname{supp}\left(w_{1}\right) \neq \emptyset$, a contradiction. This completes the proof of the fact that (3) is a consequence of (2).

Now we prove (3) implies (1). So, suppose that the four properties (3.a)(3.d) hold. We claim that if $S$ is embedded in a group then the group $S S^{-1}$ is torsion free, and thus $S$ is a positive affine semigroup. Note that in this case, $S S^{-1}$ actually is a free abelian group of rank $n-2$. Indeed, because of the assumptions there exists $u_{i}$ and $\epsilon \in\{1,2\}$ so that $u_{i} \in \operatorname{supp}\left(w_{\epsilon}\right)$ and $\operatorname{Hsupp}\left(w_{\epsilon}\right)=\emptyset$. Re-numbering the generators, if necessary, we may assume that $i=1$. Then the relation $w_{1}=w_{2}$ implies that $u_{1}=w v^{-1}$ for some $w, v \in S$ with $\operatorname{supp}(w) \cup \operatorname{supp}(v) \cup\left\{u_{1}\right\}=\operatorname{supp}\left(w_{1}\right) \cup \operatorname{supp}\left(w_{2}\right), u_{1} \notin \operatorname{supp}(w) \cup \operatorname{supp}(v)$ and $\operatorname{supp}(w) \cap \operatorname{supp}(v)=\emptyset$. It follows that

$$
S S^{-1}=\operatorname{gr}\left(u_{2}, \ldots, u_{n} \mid w_{3}\left(w v^{-1}, u_{2}, \ldots, u_{n}\right)=w_{4}\left(w v^{-1}, u_{2}, \ldots, u_{n}\right)\right) .
$$

If the second property of (3.d) holds then $\operatorname{supp}\left(w_{4}\right) \cap\left(\bigcup_{i=1}^{3} \operatorname{supp}\left(w_{i}\right)\right)=\emptyset$ and $\operatorname{Hsupp}\left(w_{4}\right)=\emptyset$. So, in particular, $u_{1} \notin \operatorname{supp}\left(w_{4}\right)$ and for $u_{k} \in \operatorname{supp}\left(w_{4}\right)$ we have that $u_{k} \notin \operatorname{supp}(w) \cup \operatorname{supp}(v) \cup \sup \left(w_{3}\right)$ and

$$
u_{k}=w_{3}\left(w v^{-1}, u_{2}, \ldots, u_{n}\right) u^{-1}
$$

with $w_{4}=u u_{k}$ and $\operatorname{supp}\left(w_{4}\right)=\operatorname{supp}(u) \cup\left\{u_{k}\right\}$. Hence we obtain that $S S^{-1}=$ $\operatorname{gr}\left(\left\{u_{2}, \ldots, u_{n}\right\} \backslash\left\{u_{k}\right\}\right)$ and this is a free abelian group of rank $n-2$, as claimed. If, on the other hand, the first property of (3.d) holds then, without loss of 
generality, we may assume that $\operatorname{supp}\left(w_{1}\right) \cap \operatorname{supp}\left(w_{3}\right) \neq \emptyset, \operatorname{Hsupp}\left(w_{2}\right)=\emptyset$ and $u_{1} \in \operatorname{supp}\left(w_{2}\right)$. So, $u_{1} \notin \operatorname{supp}\left(w_{3}\right)$. If $\operatorname{Hsupp}\left(w_{3}\right)=\emptyset$ then choose $u_{k} \in$ $\operatorname{supp}\left(w_{3}\right)$ and write $w_{3}=u_{k} v^{\prime}$ with $u_{k} \notin \operatorname{supp}\left(v^{\prime}\right)$ and $\operatorname{supp}\left(w_{3}\right)=\left\{u_{k}\right\} \cup$ $\operatorname{supp}\left(v^{\prime}\right)$. So $u_{k}=w_{4}\left(v^{\prime}\right)^{-1}$. Note that $u_{1} \notin \operatorname{supp}\left(w_{4}\right) \cup \operatorname{supp}\left(v^{\prime}\right)$. It follows that $S S^{-1}=\operatorname{gr}\left(\left\{u_{2}, \ldots, u_{n}\right\} \backslash\left\{u_{k}\right\}\right)$, a free abelian group of rank $n-2$. Finally, if $\operatorname{Hsupp}\left(w_{3}\right) \neq \emptyset$ then $\operatorname{Hsupp}\left(w_{4}\right)=\emptyset$. In this case write $w_{4}=u_{l} v^{\prime \prime}$ for some $v^{\prime \prime}$ with $u_{l} \notin \operatorname{supp}\left(v^{\prime \prime}\right)$ and $\operatorname{supp}\left(w_{4}\right)=\left\{u_{k}\right\} \cup \operatorname{supp}\left(v^{\prime \prime}\right)$. It follows that $S S^{-1}=\operatorname{gr}\left(\left\{u_{2}, \ldots, u_{n}\right\} \backslash\left\{u_{l}\right\}\right)$, again a free abelian group of rank $n-2$, as desired.

So now we show that $S$ is cancellative and thus embedded in $\mathrm{Fa}_{n-2}$. By symmetry we can assume that $\operatorname{Hsupp}\left(w_{4}\right)=\emptyset$. Then write

$$
w_{2}=y_{1}^{\gamma_{1}} \cdots y_{q}^{\gamma_{q}}, \quad w_{4}=x_{1} \cdots x_{p-1} x_{p},
$$

$\gamma_{i} \geq 1$, where $x_{1}, \ldots, x_{p}, y_{1}, \ldots, y_{q} \in\left\{u_{1}, \ldots, u_{n}\right\}$, and $\operatorname{supp}\left(w_{4}\right)$ does not intersect nontrivially the support of any other word in the defining relations.

Let $F$ be the free abelian monoid with basis $\operatorname{supp}\left(w_{1}\right) \cup\left\{y_{1}, \ldots, y_{q}\right\} \cup$ $\operatorname{supp}\left(w_{3}\right) \cup\left\{x_{1}, \ldots, x_{p-1}\right\}$. Then let $T=F / \rho$, where $\rho$ is the congruence defined by the relation $w_{1}=w_{2}$. $\operatorname{Since} \operatorname{Hsupp}\left(w_{1}\right)=\emptyset$ or $\operatorname{Hsupp}\left(w_{2}\right)=\emptyset$, we know from Proposition 2.2 that $T$ is a normal positive monoid. In particular, $T T^{-1}$ is a torsion free group. Consider the semigroup morphism

$$
f: T \times\langle u\rangle \longrightarrow T T^{-1}
$$

defined by $f(t)=t$, for $t \in T$ and $f(u)=w_{3} z^{-1}$ and $z=x_{1} \cdots x_{p-1}$. Note that $f\left(w_{3}\right)=f(z u)$. Hence the above morphism induces the following natural morphisms

$$
T \times\langle u\rangle \stackrel{\pi}{\longrightarrow}(T \times\langle u\rangle) / \nu \stackrel{\bar{f}}{\longrightarrow} T T^{-1},
$$

with $\nu$ the congruence defined by the relation $w_{3}=z u$. Put $M=(T \times\langle u\rangle) / \nu$ and note that

$$
M \cong S .
$$

For simplicity we denote $\pi(t)$ as $\bar{t}$, for $t \in T \times\langle u\rangle$. We note that $\pi_{\left.\right|_{T}}$, the restriction of $\pi$ to $T$, is injective. Indeed, suppose $s, t \in T$ are such that $\pi(s)=$ $\pi(t)$. Then

$$
s-t \in K[T \times\langle u\rangle]\left(z u-w_{3}\right),
$$

an ideal in $K[T \times\langle u\rangle]$. So, $s-t=\alpha\left(z u-w_{3}\right)$, for some $\alpha \in K[T \times\langle u\rangle]$. Now $K[T \times\langle u\rangle]$ has a natural $\mathbb{N}$-gradation, with respect to the degree in $u$. Clearly, $s-t$ and $w_{3}$ have degree zero. Let $\alpha_{h}$ be the highest degree term of $\alpha$ with respect to this gradation. Then,

$$
0=\alpha_{h} z u
$$

Since $T \times\langle u\rangle$ is contained in a torsion free group, we know that $K[T \times\langle u\rangle]$ is a domain. So we get that $\alpha_{h}=0$ and thus $\alpha=0$. Hence $s=t$ and therefore indeed $\pi_{\mid T}$ is injective. So we will identify the element $\pi(t)$ with $t$, for $t \in T$. 
Next we note that $\bar{u}$ is a cancellable element in $M$. Indeed, let $\bar{x}, \bar{y} \in M$ and suppose $\bar{u} \bar{x}=\bar{u} \bar{y}$. This means that

$$
u x-u y \in K[T \times\langle u\rangle]\left(u z-w_{3}\right),
$$

i.e.

$$
u x-u y=\alpha\left(u z-w_{3}\right)
$$

for some $\alpha \in K[T \times\langle u\rangle]$, where $x, y \in T \times\langle u\rangle$ are inverse images of $\bar{x}, \bar{y}$. Again consider the $\mathbb{N}$-gradation on $K[T \times\langle u\rangle]$ via the degree in $u$. Let $\alpha_{0}$ be the zero degree component of $\alpha$. Then it follows that

$$
0=\alpha_{0} w_{3}
$$

Hence $\alpha_{0}=0$, as $K[T]$ is a domain, and thus

$$
\alpha \in K[T \times\langle u\rangle] u .
$$

Using again that $K[T \times\langle u\rangle]$ is a domain, we get from (2) that

$$
x-y \in K[T \times\langle u\rangle]\left(u z-w_{3}\right) .
$$

Hence $\bar{x}=\bar{y} \in M$, as desired.

In the above we thus have shown that $\bar{u}$ is cancellable in $M$. Hence $x_{p}$ is cancellable in $S$. The argument of the proof holds for all elements $x_{1}, \ldots, x_{p}$. So, all elements $x_{1}, \ldots, x_{p}$ are cancellable in $S$. By a similar argument, if $\operatorname{Hsupp}\left(w_{2}\right)=\emptyset$, this also holds for all elements $y_{i} \in \operatorname{supp}\left(w_{2}\right) \backslash \operatorname{supp}\left(w_{3}\right)$.

On the other hand, if $\operatorname{Hupp}\left(w_{2}\right) \neq \emptyset$ and thus $\operatorname{Hsupp}\left(w_{1}\right)=\emptyset$, then similarly one shows that $u_{i}$ is cancellable in $S$, for every $u_{i} \in \operatorname{supp}\left(w_{1}\right) \backslash \operatorname{supp}\left(w_{3}\right)$. Clearly, $S$ is contained in its localization $S_{C}$, with respect to the multiplicatively closed set of the cancellable elements. In view of the form of the defining relations of $S$, this implies that $S_{C}$ is a group. So $S$ is a cancellative monoid in $S S^{-1}=\mathrm{Fa}_{n-2}$.

Finally, we show that $S$ is normal, by proving it is a union of finitely many finitely generated free abelian monoids. To so, note that conditions (3.a)-(3.d) imply that $\operatorname{Hsupp}\left(w_{i}\right)=\emptyset$ and $\operatorname{Hsupp}\left(w_{j}\right)=\emptyset$ for some $i \in\{1,2\}$ and $j \in\{3,4\}$. Furthermore, $\operatorname{supp}\left(w_{i}\right) \cap \operatorname{supp}\left(w_{l}\right)=\emptyset$ for all $l$ with $l \neq i$, or $\operatorname{supp}\left(w_{j}\right) \cap$ $\operatorname{supp}\left(w_{l}\right)=\emptyset$ for all $l$ with $l \neq j$. Without loss of generality we may assume the former holds. Note that if $w_{k}=u_{q}$ for some $k$ and some $q$ then the assertion follows from Proposition 2.2. Hence, without loss of generality, we may assume that $\left|\operatorname{supp}\left(w_{k}\right)\right|>1$ for $k=1,2,3,4$.

$\operatorname{Because} \operatorname{Hsupp}\left(w_{i}\right)=\emptyset$, it is easily seen, using the relation involving $w_{i}$, that $s$ can be written as a product of elements of $\left\{u_{1}, \ldots, u_{n}\right\} \backslash\{u\}$ for some $u \in \operatorname{supp}\left(w_{i}\right)$. If not all elements of $\operatorname{supp}\left(w_{j}\right)$ occur in this product of $s$, then $s \in\left\langle\left\{u_{1}, \ldots, u_{n}\right\} \backslash\{u, v\}\right\rangle$, with $v \in \operatorname{supp}\left(w_{j}\right)$. Now because of the defining relations one easily sees that $\left\langle\left\{u_{1}, \ldots, u_{n}\right\} \backslash\{u, v\}\right\rangle$ is a free abelian monoid, as desired. If, on the other hand, all elements of $\operatorname{supp}\left(w_{j}\right)$ occur in the expression of $s$ then, using the relation involving $w_{j}$ (several times if needed) and using the 
fact that $\operatorname{supp}\left(w_{i}\right) \cap \operatorname{supp}\left(w_{l}\right)=\emptyset$ for all $l \neq i$, we can reduce to the previous case. This ends the proof.

As a matter of example, it follows at once from Theorem 3.1 that the commutative algebra $K\left\langle u_{1}, u_{2}, u_{3}, u_{4}, u_{5} \mid u_{1} u_{2}=u_{3}^{2}, u_{1} u_{3}=u_{4} u_{5}\right\rangle$ is a normal domain, while the commutative algebra $K\left\langle u_{1}, u_{2}, u_{3}, u_{4} \mid u_{1} u_{2}=u_{3}^{2}, u_{1} u_{3}=u_{4}^{2}\right\rangle$ is a domain that is not normal.

Finally, we describe the class group of positive monoid defined by two relations. We use the same notation as in the proof of Theorem 3.1. If $\left(\operatorname{supp}\left(w_{1}\right) \cup\right.$ $\left.\operatorname{supp}\left(w_{2}\right)\right) \cap\left(\operatorname{supp}\left(w_{3}\right) \cup \operatorname{supp}\left(w_{4}\right)\right)=\emptyset$ then $S \cong S_{1} \times S_{2}$, with $S_{1}=\left\langle\operatorname{supp}\left(w_{1}\right) \cup\right.$ $\operatorname{supp}\left(w_{2}\right)\left|w_{1}=w_{2}\right\rangle$ and $S_{2}=\left\langle\operatorname{supp}\left(w_{3}\right) \cup \operatorname{supp}\left(w_{4}\right) \mid w_{3}=w_{4}\right\rangle$. Clearly, in this case, $\operatorname{cl}(S) \cong \operatorname{cl}\left(S_{1}\right) \times \operatorname{cl}\left(S_{2}\right)$, and the result follows from Theorem 2.4. So, assume $S$ satisfies one of the properties in condition (3.d) in Theorem 3.1 Then, we can write

$$
S=\left\langle u_{1}, \ldots, u_{n}, \ldots, u_{m}\right\rangle
$$

with relations

$$
\begin{aligned}
u_{1} \cdots u_{k_{1}} u_{k_{2}+1} \cdots u_{k_{3}} & =u_{k_{1}+1}^{a_{k_{1}+1}} \cdots u_{k_{2}}^{a_{k_{2}}} u_{k_{3}+1}^{a_{k_{3}+1}} \cdots u_{k_{4}}^{a_{k_{4}}} \\
u_{1}^{a_{1}} \cdots u_{k_{1}}^{a_{k_{1}}} u_{k_{1}+1}^{b_{k_{1}+1}} \cdots u_{k_{2}}^{b_{k_{2}}} u_{k_{4}+1}^{a_{k_{4}+1}} \cdots u_{k_{5}}^{a_{k_{5}}} & =u_{k_{5}+1} \cdots u_{n},
\end{aligned}
$$

with $0<k_{1} \leq k_{2} \leq k_{3} \leq k_{4} \leq k_{5}<n \leq m$ and all $a_{i}, b_{j} \geq 1$ and (we agree that if $k_{1}=k_{2}, k_{2}=k_{3}, k_{3}=k_{4}$ or $k_{4}=k_{5}$ then the factors $u_{k_{1}+1}^{a_{k_{1}+1}} \cdots u_{k_{2}}^{a_{k_{2}}}$, $u_{k_{1}+1}^{b_{k_{1}+1}} \cdots u_{k_{2}}^{b_{k_{2}}}, u_{k_{2}+1} \cdots u_{k_{3}}, u_{k_{3}+1}^{a_{k_{3}+1}} \cdots u_{k_{4}}^{a_{k_{4}}}$, or $u_{k_{4}+1}^{a_{k_{4}+1}} \cdots u_{k_{5}}^{a_{k_{5}}}$ are the empty words). So, the two cases discussed in condition (3.d) of Theorem 3.1 correspond to $k_{1}=k_{2}$ and $k_{1}<k_{2}$, respectively.

As in the previous section, in order to compute the class group, we also may assume that $n=m$. Moreover, we may assume that $w_{i} \notin\left\{u_{1}, \ldots, u_{n}\right\}$ for $i=1,2,3,4$, as otherwise $S$ can be presented by a single relation and then the class group is given in Theorem 2.4. Under this restriction, in the next lemma, we describe the principal ideals as divisorial products of minimal prime ideals. Note that there are two possible types of minimal primes in $S$. First, there are

$$
Q=\left(u_{i}, u_{j}\right),
$$

where $u_{i}$ and $u_{j}$ each belong to the support of different sides of one of the defining relations and do not belong to the supports of the words in the other relation. To prove that $Q$ is a prime ideal we may assume, by symmetry, that $u_{i}, u_{j} \in \operatorname{supp}\left(w_{1}\right) \cup \operatorname{supp}\left(w_{2}\right)$. Clearly, $S / Q$ is then generated by the natural images of the elements $u_{q}, q \neq i, j$, subject to the unique relation $w_{3}=w_{4}$. Since $u_{i}, u_{j} \notin \operatorname{supp}\left(w_{3}\right) \cup \operatorname{supp}\left(w_{4}\right)$, it is easily seen that $(S / Q) \backslash\{0\}$ is a multiplicatively closed set, as desired. Second, there are minimal primes of the form

$$
Q=\left(u_{i}, u_{j}, u_{k}\right)
$$

where $u_{i}$ belongs to the support of a word in each of the two relations, $u_{j}$ and $u_{k}$ belong to the support of a word in a defining relation but on a different 
side than $u_{i}$, and furthermore $u_{j}$ and $u_{k}$ are involved in different relations. In particular, $j \neq k$. Clearly, existence (and the number) of minimal primes of the latter type depends on the existence of strict inequalities $k_{i}<k_{i+1}$.

The formulas obtained in the following Lemma 3.2 should be interpreted in such a way that principal ideals $S u_{w}$ and primes $P_{y, z}$ or $P_{t, v, x}$ are deleted if some index does not occur in the defining relations. So, for example $P_{y, k_{3}+1}$ is not defined and hence ignored if $k_{3}=k_{4}$.

\section{Lemma 3.2 Let}

$$
\begin{aligned}
S=\left\langle u_{1}, \ldots, u_{n} \quad\right| & u_{1} \cdots u_{k_{1}} u_{k_{2}+1} \cdots u_{k_{3}}=u_{k_{1}+1}^{a_{k_{1}+1}} \cdots u_{k_{2}}^{a_{k_{2}}} u_{k_{3}+1}^{a_{k_{3}+1}} \cdots u_{k_{4}}^{a_{k_{4}}} \\
& \left.u_{1}^{a_{1}} \cdots u_{k_{1}}^{a_{k_{1}}} u_{k_{1}+1}^{b_{k_{1}+1}} \cdots u_{k_{2}}^{b_{k_{2}}} u_{k_{4}+1}^{a_{k_{4}+1}} \cdots u_{k_{5}}^{a_{k_{5}}}=u_{k_{5}+1} \cdots u_{n}\right\rangle,
\end{aligned}
$$

with $0<k_{1} \leq k_{2} \leq k_{3} \leq k_{4} \leq k_{5}<n$ and all $a_{i}, b_{j} \geq 1$, be a normal monoid that cannot be presented with a single relation. Put $P_{y, z}$, the minimal prime ideal of $S$ generated by $\left\{u_{y}, u_{z}\right\}, y \in\left\{k_{2}+1, \ldots, k_{3}\right\}, z \in\left\{k_{3}+1, \ldots, k_{4}\right\}$ or $y \in\left\{k_{4}+1, \ldots, k_{5}\right\}, z \in\left\{k_{5}+1, \ldots, n\right\}$ and put $P_{t, v, x}$, the minimal prime ideal of $S$ that is generated by $\left\{u_{t}, u_{v}, u_{x}\right\}, t \in\left\{1, \ldots, k_{1}\right\}, v \in\left\{k_{1}+1, \ldots, k_{2}, k_{3}+\right.$ $\left.1, \ldots, k_{4}\right\}, x \in\left\{k_{5}+1, \ldots, n\right\}$ or $t \in\left\{k_{2}+1, \ldots, k_{3}\right\}, v \in\left\{k_{1}+1, \ldots, k_{2}\right\}$, $x \in\left\{k_{5}+1, \ldots, n\right\}$. Then

1. $S u_{w}=\left(\prod_{l=k_{5}+1}^{n}\left(\prod_{m=1}^{k_{1}} P_{m, w, l} \prod_{m=k_{2}+1}^{k_{3}} P_{m, w, l}\right)\right)^{*}$, for $w \in\left\{k_{1}+1, \ldots\right.$, $\left.k_{2}\right\}$,

2. $S u_{w}=\left(\prod_{l=k_{5}+1}^{n}\left(\prod_{m=1}^{k_{1}} P_{m, w, l}\right)\right)^{*} *\left(\prod_{m=k_{2}+1}^{k_{3}} P_{m, w}\right)^{*}$, for $w \in\left\{k_{3}+\right.$ $\left.1, \ldots, k_{4}\right\}$

3. $S u_{w}=\left(\prod_{l=k_{5}+1}^{n} P_{w, l}\right)^{*}$, for $w \in\left\{k_{4}+1, \ldots, k_{5}\right\}$,

4. $S u_{w}=\left(\prod_{l=k_{5}+1}^{n}\left(\prod_{m=k_{1}+1}^{k_{2}} P_{w, m, l}^{a_{m}} \prod_{m=k_{3}+1}^{k_{4}} P_{w, m, l}^{a_{m}}\right)\right)^{*}$, for $w \in\{1, \ldots$, $\left.k_{1}\right\}$,

5. $S u_{w}=\left(\prod_{l=k_{5}+1}^{n}\left(\prod_{m=k_{1}+1}^{k_{2}} P_{w, m, l}^{a_{m}}\right)\right)^{*} *\left(\prod_{m=k_{3}+1}^{k_{4}} P_{w, m}^{a_{m}}\right)^{*}$, for $w \in$ $\left\{k_{2}+1, \ldots, k_{3}\right\}$,

6. $S u_{w}=\left(\prod_{l=1}^{k_{1}}\left(\prod_{m=k_{1}+1}^{k_{2}} P_{l, m, w}^{a_{m}} \prod_{m=k_{3}+1}^{k_{4}} P_{l, m, w}^{a_{m}}\right)^{a_{l}}\right)^{*} *$ $\left(\prod_{m=k_{1}+1}^{k_{2}}\left(\prod_{l=1}^{k_{1}} P_{l, m, w} \prod_{l=k_{2}+1}^{k_{3}} P_{l, m, w}\right)^{b_{m}}\right)^{*} *\left(\prod_{l=k_{4}+1}^{k_{5}} P_{l, w}^{a_{l}}\right)^{*}$,

for $w \in\left\{k_{5}+1, \ldots, n\right\}$,

Proof. For $w \in\{1, \ldots, n\}$, one notices that in the expressions for $S_{w}$, in the statement of the lemma, precisely all the minimal primes $P$ occur that contain $u_{w}$. Using the defining relations one then easily verifies, as in the proof of Lemma 2.3. that the proposed formulae hold in the localizations $S_{P}$. Hence the result follows. 
Our next aim is to describe the class group of $S$. Surprisingly, the proof is obtained by a reduction to the case considered in Theorem 2.4. The definitions of $d_{1}$ and $d_{2}$ in the following result should again be interpreted in the correct way when some $k_{i}=k_{i+1}$. We agree to ignore all $a_{i}$ (respectively, $b_{j}$ ) for which $u_{i}$ (respectively $u_{j}$ ) does not occur in the defining relations.

Theorem 3.3 Let

$$
\begin{aligned}
& S=\left\langle u_{1}, \ldots, u_{n}, \ldots, u_{m}\right| u_{1} \cdots u_{k_{1}} u_{k_{2}+1} \cdots u_{k_{3}}=u_{k_{1}+1}^{a_{k_{1}+1}} \cdots u_{k_{2}}^{a_{k_{2}}} u_{k_{3}+1}^{a_{k_{3}+1}} \cdots u_{k_{4}}^{a_{k_{4}}} \\
& \left.\qquad u_{1}^{a_{1}} \cdots u_{k_{1}}^{a_{k_{1}}} u_{k_{1}+1}^{b_{k_{1}+1}} \cdots u_{k_{2}}^{b_{k_{2}}} u_{k_{4}+1}^{a_{k_{4}+1}} \cdots u_{k_{5}}^{a_{k_{5}}}=u_{k_{5}+1} \cdots u_{n}\right\rangle \\
& \text { (with } \left.0<k_{1} \leq k_{2} \leq k_{3} \leq k_{4} \leq k_{5}<n \leq m \text { and all } a_{i}, b_{j} \geq 1\right) \text { be a normal } \\
& \text { positive monoid that does not admit a presentation with a single defining rela- } \\
& \text { tion. Let } Q=\left\{a_{t} a_{v}+b_{v} \mid t \in\left\{1, \ldots, k_{1}\right\}, v \in\left\{k_{1}+1, \ldots, k_{2}\right\}\right\} \cup\left\{a_{t} a_{v} \mid t \in\right. \\
& \left.\left\{1, \ldots, k_{1}\right\}, v \in\left\{k_{3}+1, \ldots, k_{4}\right\}\right\} \cup\left\{a_{y} \mid y \in\left\{k_{4}+1, \ldots, k_{5}\right\}\right\} \text {. Then } \\
& \qquad \operatorname{cl}(K[S]) \cong \operatorname{cl}(S) \cong \mathbb{Z}^{f} \times\left(\mathbb{Z}_{d_{1}}\right)^{k_{1}+k_{3}-k_{2}-1} \times\left(\mathbb{Z}_{d_{2}}\right)^{n-k_{5}-1}
\end{aligned}
$$

where

$$
\begin{aligned}
f= & \left(k_{3}-k_{2}\right)\left(k_{4}-k_{3}\right)+\left(k_{5}-k_{4}\right)\left(n-k_{5}\right)+k_{1}\left(k_{4}-k_{3}+k_{2}-k_{1}\right)\left(n-k_{5}\right) \\
& +\left(k_{3}-k_{2}\right)\left(k_{2}-k_{1}\right)\left(n-k_{5}\right)-(n-2),
\end{aligned}
$$

with

$$
d_{1}=\operatorname{gcd}\left(a_{k_{1}+1}, \ldots, a_{k_{2}}, a_{k_{3}+1}, \ldots, a_{k_{4}}\right)
$$

and

$$
d_{2}=\left\{\begin{array}{ll}
\operatorname{gcd}\left(a_{1} d_{1}, \ldots, a_{k_{1}} d_{1}, b_{k_{1}+1}, \ldots, b_{k_{2}}, a_{k_{4}+1}, \ldots, a_{k_{5}}\right) & \text { if } k_{2}<k_{3} \\
\operatorname{gcd}(q \mid q \in Q) & \text { if } k_{2}=k_{3}
\end{array} .\right.
$$

Proof. As mentioned earlier, withou loss of generality we may assume that $n=m$. It is shown in the proof of Theorem 3.1 that $S S^{-1} \cong \mathrm{Fa}_{n-2}$, the free abelian group of rank $n-2$. Because $\mathrm{U}(S)=\{1\}$, we get that $P(S)$ and $S S^{-1}$ are isomorphic, and thus they have the same torsion free rank. Since the torsion free rank of $\operatorname{cl}(S)$ is the difference of the torsion free rank of $D(S)$ and the torsion free rank of $P(S)$, to establish the description of the torsion free part of $\operatorname{cl}(S)$, we only need to show that there are $\left(k_{3}-k_{2}\right)\left(k_{4}-k_{3}\right)+\left(k_{5}-\right.$ $\left.k_{4}\right)\left(n-k_{5}\right)+k_{1}\left(k_{4}-k_{3}+k_{2}-k_{1}\right)\left(n-k_{5}\right)+\left(k_{3}-k_{2}\right)\left(k_{2}-k_{1}\right)\left(n-k_{5}\right)$ minimal primes in $S$. But this easily follows from the description of the minimal primes given Lemma 3.2

As in the proof of Theorem 2.4, we consider $\operatorname{cl}(S)$ as a finitely generated $\mathbb{Z}$-module, so that its presentation is determined by an integer matrix $M$ of size $r \times n$, where $r$ is the number of minimal primes in $S$, hence the basis of $D(S)$. Therefore, the rows are indexed by all triples $(t, v, x)$ and all pairs $(y, z)$, as described in Lemma 3.2. We agree on the following ordering of the set of rows of $M$ : all triples $(t, v, x)$ are ordered lexicographically, so are all the pairs $(y, z)$ and $(t, v, x)<(y, z)$ for every $t, v, x, y, z$. The columns are indexed by 
$1,2, \ldots, n$, where the $i$-th column corresponds to the generator $S u_{i}$, written as a vector in terms of the minimal primes of $S$, as in Lemma 3.2

We consider the block decomposition of $M$ determined by the following partitions of the sets $C$ and $R$ of columns and rows:

$$
C=D_{1} \cup D_{2},
$$

where $D_{1}=\left\{1, \ldots, k_{5}\right\}$ and $D_{2}=\left\{k_{5}+1, \ldots, n\right\}$. Notice that $\left|D_{2}\right| \geq 2$ because $S$ does not admit a presentation with one defining relation. Let $R_{0}=\{(y, z) \mid$ $\left.y \in\left\{k_{2}+1, \ldots, k_{3}\right\}, z \in\left\{k_{3}+1, \ldots, k_{4}\right\}\right\}, R_{y}=\left\{(y, z) \mid z \in\left\{k_{5}+1, \ldots, n\right\}\right\}$ for $y \in\left\{k_{4}+1, \ldots, k_{5}\right\}$. For every triple $(t, v, x)$ we also define $R_{t, v}=\{(t, v, x) \mid$ $\left.x \in\left\{k_{5}+1, \ldots, n\right\}\right\}$. Then

$$
R=\bigcup R_{t, v} \cup R_{0} \cup \bigcup_{y=k_{2}+1}^{k_{3}} R_{y},
$$

where the first union runs over all pairs $(t, v)$ such that the set $R$ of rows contains a triple of the form $(t, v, x)$.

Consider any of the block submatrices $M_{R_{t, v}, C}$ or $M_{R_{y}, C}$, with $R_{t, v}, R_{y}$ as above. From Lemma 3.2 it follows that, ignoring the zero columns of this submatrix, it has the form

$$
\left(\begin{array}{cccccc}
a & b & d & 0 & \cdots & 0 \\
a & b & 0 & d & \cdots & 0 \\
\cdots & \cdots & \cdots & \cdots & \cdots & 0 \\
a & b & 0 & \cdots & 0 & d
\end{array}\right),
$$

for some $a, b$ such that either $a=1$ or $b=1$ and for some $d$. Here the columns of the scalar matrix determined by $d$ are indexed by $D_{2}$. So, subtracting the first row in each such block $\left(M_{R_{t, v}, C}\right.$ or $\left.M_{R_{y}, C}\right)$ from all the remaining rows in this block and next subtracting the last $n-\left(k_{5}+1\right)$ columns of the entire matrix from column $k_{5}+1$, we get a matrix $M^{\prime}$ such that each block $M_{X, D_{1}}^{\prime}$, for $X=R_{y}$ or $X=R_{t, v}$, has only the first row nonzero and $M_{R, D_{2}}^{\prime}=M_{R, D_{2}}$. Moreover $M_{R_{0}, C}^{\prime}=M_{R_{0}, C}$. Therefore, the nonzero entries of the last column of $M^{\prime}$ are the only nonzero entries in their respective rows. Denote by $Y$ the set of all such rows of $M^{\prime}$. Then these nonzero entries (in the last column of $M^{\prime}$ ), and with our convention as explained before the theorem, are:

$$
\begin{array}{cllll}
a_{t} a_{v}+b_{v} & \text { for } & t \in\left\{1, \ldots, k_{1}\right\}, v \in\left\{k_{1}+1, \ldots, k_{2}\right\} & \text { if } & k_{1} \neq k_{2} \\
a_{t} a_{v} & \text { for } & t \in\left\{1, \ldots, k_{1}\right\}, v \in\left\{k_{3}+1, \ldots, k_{4}\right\} & \text { if } & k_{3} \neq k_{4} \\
b_{t} & \text { for } & t \in\left\{k_{1}+1, \ldots, k_{2}\right\} & \text { if } & k_{1} \neq k_{2}, k_{2} \neq k_{3} \\
a_{y} & \text { for } & y \in\left\{k_{4}+1, \ldots, k_{5}\right\} & \text { if } & k_{4} \neq k_{5} .
\end{array}
$$

Notice that the greatest common divisor of the specified set of elements is equal to $d_{2}$, as defined in the statement of the theorem. Thus, row elimination within the block $M_{Y, C}^{\prime}$ allows us to produce a row of the form $\left(0, \ldots, 0, d_{2}\right)$ and replace all other rows of $M_{Y, C}^{\prime}$ by zero rows. The same argument can be applied to the 
nonzero entries in the subsequent columns: $n-1, n-2, \ldots, k_{5}+2$. This leads to a matrix $M^{\prime \prime}$ (of the same size as the original matrix $M$ ) with $n-k_{5}-1$ rows of the form $\left(0, \ldots, d_{2}, 0, \ldots, 0\right)$, with $d_{2}$ in positions $k_{5}+2, \ldots, n$, with no other nonzero entries in their respective columns. So, it remains to find the normal form of the matrix $N$ obtained by deleting in $M^{\prime \prime}$ the last $n-k_{5}-1$ columns and the rows that contain the nonzero entries in these columns. It is easy to see that the last column of $N$ is a $\mathbb{Z}$-combination of the remaining columns. Namely, we have $C_{k_{5}+1}=a_{1} C_{1}+\cdots+a_{k_{1}} C_{k_{1}}+b_{k_{1}+1} C_{k_{1}+1}+\cdots+b_{k_{2}} C_{k_{2}}+$ $a_{k_{4}+1} C_{k_{4}+1}+\cdots+a_{k_{5}} C_{k_{5}}$. Hence by column operations we can make this column zero. Then, deleting this column, we get a matrix with $k_{4}$ columns that is of he form $\left(\begin{array}{cc}N^{\prime} & 0 \\ 0 & I\end{array}\right)$ for a matrix $N^{\prime}$ and the identity $\left(k_{5}-k_{4}\right) \times\left(k_{5}-k_{4}\right)$ matrix $I$. It is easy to see that $N^{\prime}$ corresponds to the monoid $T$ with the presentation $u_{1} \cdots u_{k_{1}} u_{k_{2}+1} \cdots u_{k_{3}}=u_{k_{1}+1}^{a_{k_{1}+1}} \cdots u_{k_{2}}^{a_{k_{2}}} u_{k_{3}+1}^{a_{k_{3}+1}} \cdots u_{k_{4}}^{a_{k_{4}}}$ and with the generating set $u_{1}, \ldots, u_{k_{4}}$. Hence, by Theorem $2.4 \operatorname{cl}(T)=\mathbb{Z}^{e} \times \mathbb{Z}_{d_{1}}^{k_{3}-k_{2}+k_{1}-1}$, where $e=\left(k_{1}+k_{3}-k_{2}\right)\left(k_{2}-k_{1}+k_{4}-k_{3}\right)-\left(k_{4}-1\right)$. Therefore, the normal form of $M$ has $k_{3}-k_{2}+k_{1}-1$ copies of $d_{1}$ and $n-k_{5}-1$ copies of $d_{2}$ and a certain number of entries equal to 1 . By the comment at the beginning of the proof, it must have $f$ zero rows. Hence, the result follows.

Acknowledgments This research was supported by the Onderzoeksraad of Vrije Universiteit Brussel, Fonds voor Wetenschappelijk Onderzoek (Flanders), Flemish-Polish bilateral agreement BIL2005/VUB/06 and a MNiSW research grant N201 004 32/0088 (Poland). The first author was also funded by a Ph.D grant of the Institute for the Promotion of Innovation through Science and Technology in Flanders (IWT-Vlaanderen).

The authors are grateful to the referee for making several valuable comments and suggestions. This resulted in a completely revised format of an earlier version of the paper.

\section{References}

[1] W. Bruns and J. Gubeladze, Semigroup algebras and discrete geometry, Séminaire \& Congrès 6 (2002), Soc. Math. France, Paris, 43-127.

[2] W. Bruns and J. Herzog, Cohen - Macaulay Rings, Cambridge Univ. Press, rev. ed., Cambridge, 1998.

[3] S.T. Chapman, P.A. Garcia-Sánchez, D. Llena and J.C. Rosales, Presentations of finitely generated cancellative abelian monoids and nonnegative solutions of systems of linear equations, Discrete Appl. Math. 154 (2006), 1947-1959.

[4] L.G. Chouinard II., Krull semigroups and divisor class groups, Canad. J. Math. 23 (1981), 1459-1468.

[5] P. Dueck, S. Hosten and B. Sturmfels, Normal toric ideals of low codimension, arXiv:0801.3826, preprint. 
[6] P. Etingof, T. Schedler and A. Soloviev, Set-theoretical solutions to the quantum Yang-Baxter equation, Duke Math. J. 100 (1999), 169-209.

[7] R. Fossum, The Divisor Class Group of a Krull Domain, Springer-Verlag, New York, 1973.

[8] T. Gateva-Ivanova and M. Van den Bergh, Semigroups of $I$-type, J. Algebra 206 (1998), 97-112.

[9] R. Gilmer, Commutative Semigroup Rings, Univ. Chicago Press, Chicago, 1984.

[10] I. Goffa, E. Jespers, Monoids of IG-type and maximal orders, J. Algebra 308 (2007), 44-62.

[11] I. Goffa, E. Jespers and J. Okniński, Primes of height one and a class of Noetherian finitely presented algebras, Internat. J. Algebra Comput. 17 (2007), 1465-1491.

[12] E. Jespers and J. Okniński, Noetherian Semigroup Algebras, Algebra and Applications, Springer, 2007.

[13] E. Miller and B. Sturmfels, Combinatorial Commutative Algebra, Graduate Texts in Mathematics 227, Springer, 2005.

[14] H. Ohsugi and T. Hibi, Toric ideals arising form contingency tables, in: Commutative algebra and combinatorics. Part I: Computational algebra and combinatorics of toric ideals. Part II: Topics in commutative algebra and combinatorics. Ramanujan Mathematical Society Lecture Notes Series 4, pp. 91-115, 2007.

[15] J.C. Rosales and P.A. Garcia-Sánchez, On normal affine semigroups, Linear Algebra Appl. 286 (1999), 175-186.

[16] A. Simis and R.H. Villareal, Constraints for the normality of monomial subrings and birationality, Proc AMS 131 (2002), 2043-2048.

[17] B. Sturmfels, Gröbner Bases and Convex Polytopes, Univ. Lect. Ser. 8, Amer. Math. Soc., 1996.

[18] R.H. Villareal, Monomial Algebras, Marcel Dekker, 2001.

I. Goffa and E. Jespers

Department of Mathematics

Vrije Universiteit Brussel

Pleinlaan 2

1050 Brussel, Belgium

efjesper@vub.ac.be and igoffa@vub.ac.be
J. Okniński

Institute of Mathematics

Warsaw University

Banacha 2

02-097 Warsaw, Poland

okninski@mimuw.edu.pl 